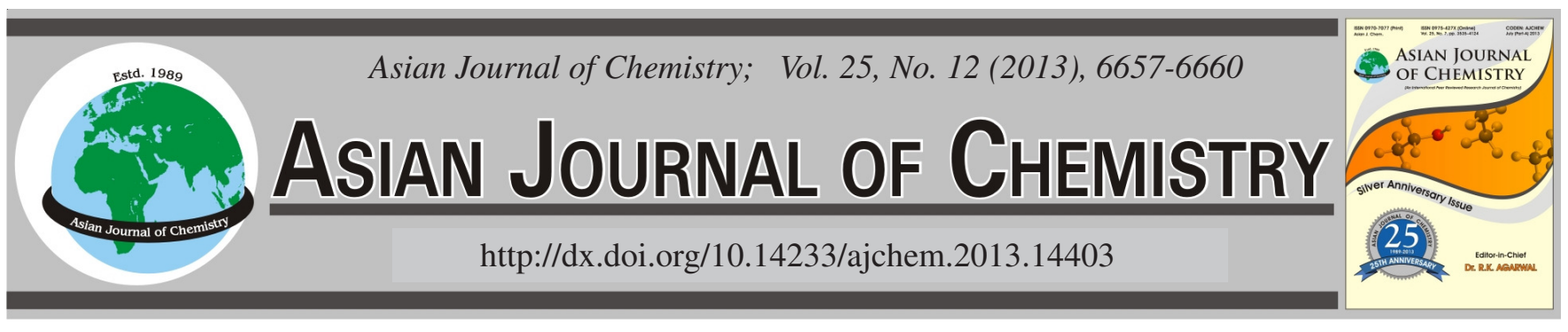

\title{
Determination of the Critical Micelle Concentration of Sodium Dodecyl Sulphate in Aqueous Solution in the Presence of an Organic Additive of Acetonitrile by Conductometry and an Inorganic Additive of Phosphate by Fluorometry
}

\author{
YuguO YANG ${ }^{1}$, QiAn Zhang ${ }^{2}$, Tianlin $\mathrm{WANG}^{2, *}$ and YANLAN YANG ${ }^{2}$
}

${ }^{1}$ School of Science, Beijing Jiaotong University, No. 3 Shang Yuan Cun, Xizhimen Wai, Beijing 100044, P.R. China

${ }^{2}$ Department of Chemistry, Shanghai University, No. 99 Shangda Road, Shanghai 200444, P.R. China

*Corresponding author: E-mail: zhxh401@yahoo.com.cn

(Received: 7 August 2012;

Accepted: 27 May 2013)

AJC-13542

\begin{abstract}
The critical micelle concentration (CMC) is the single most useful quantity for characterizing surfactants and the most significant parameter when working with ionic micellar phases, such as micellar electrokinetic chromatography (MEKC). Sodium dodecyl sulphate (SDS) was the first and is the most popular surfactant used in MEKC. Acetonitrile is often used as an organic additive for selectivity adjustment of solutes and solubility enhancement of hydrophobic compounds and phosphate as an inorganic additive for obtaining desired $\mathrm{pH}$ and buffer capacity in MEKC. The effect of acetonitrile on the CMC of SDS was studied by conductometry and the effect of phosphate buffer on the CMC of SDS in acetonitrile-water binary solvent was studied by fluorometry with the use of pyrene as a probe. It was found that the CMC of SDS first decreased up to $3 \%(\mathrm{v} / \mathrm{v})$ and then increased with increasing of the volume ratio of acetonitrile to water up to $5 \%$ $(\mathrm{v} / \mathrm{v})$. The minimum of the CMC of SDS in acetonitrile-water binary solvent was found at the volume ratio of $3 \%(\mathrm{v} / \mathrm{v})$ acetonitrile to water and was determined to be $7.7 \mathrm{mM}$. The CMC value of SDS in acetonitrile-water (3\%,v/v) binary solvent decreased with increasing of phosphate in the concentration range of $10-50 \mathrm{mM}$. The information provided in this paper is expected to be useful for optimization of separation and interpretation of migration behaviour of solutes in MEKC.
\end{abstract}

Key Words: Critical micelle concentration, Sodium dodecyl sulphate, Acetonitrile, Phosphate, Conductometry, Fluorometry.

L -

\section{INTRODUCTION}

Surfactants, some times called surface-active agents, are amphiphilic molecules with distinct hydrophobic and hydrophilic regions in their chemical structures. Depending on their chemical structures they can be nonionic, cationic, anionic, or zwitter ionic. Surfactants have been extensively used in various analytical techniques ${ }^{1-3}$. Among many applications, micellar electrokinetic chromatography (MEKC) ${ }^{4,5}$ and cloud point extraction $(\mathrm{CPE})^{6,7}$ are respective examples of successful applications of ionic and non-ionic surfactants in analytical chemistry.

In polar solvents, surfactant molecules arrange themselves into organized molecular assemblies known as micelles due to their amphiphilic character. The hydrophobic part of the aggregate forms the core of the micelle, while the polar head groups are located at the micelle-water interface in contact with and hydrated by a number of water molecules. The concentration (actually a narrow range) above which micelles form is called the critical micelle concentration (CMC). Above the $\mathrm{CMC}$, monomers and micelles exist in dynamic equilibrium. In most cases, application of surfactants in analytical chemistry makes use of the distinctive features of the surfactants: micelle formation and solubilization of water-insoluble substances by micelles. Therefore, the critical micelle concentration (CMC) is the single most useful quantity for characterizing surfactants and the most significant parameter when working with ionic micellar phases, such as MEKC. Among the separation techniques, MEKC is most powerful one since it allows the separation of mixtures of both ionized and neutral compounds. The separation is achieved due to different partition of analytes between the polar (usually aqueous) and micellar phases ${ }^{4,5}$. Thus, to optimize the separation in MEKC it would be very useful to know the CMC of the surfactant used under the experimental condition.

Sodium dodecyl sulphate (SDS) was the first ${ }^{4}$ and is the most popular surfactant used in $\mathrm{MEKC}^{8,9}$. Acetonitrile is often added as an organic additive for selectivity adjustment of solutes and solubility enhancement of hydrophobic compounds and phosphate as inorganic additive for obtaining desired $\mathrm{pH}$ and buffer capacity in $\mathrm{MEKC}^{10-16}$. Additives can cause the CMC value of SDS different from that determined in pure water ${ }^{17-20}$. Bosch et al. ${ }^{20}$ determined the CMC values of SDS in aqueous 
unbuffered and buffered systems with different concentrations of phosphate at $\mathrm{pH} 7$ by using four methodologies (conductometry, conductometric titration, spectrophotometric titration and MEKC). Jacquier and Desbene ${ }^{21}$ studied the effect of electrolytes on the CMC values of SDS in aqueous solution and the effect of organic solvents on the CMC values of SDS in buffered aqueous solution with borax at $5 \mathrm{mM}$ and $\mathrm{pH} 9.2$. Chen et $a .^{22}$ determined the CMC value of SDS in acetonitrilewater $(2.5 \%, \mathrm{v} / \mathrm{v})$ binary solvent containing $25 \mathrm{mM} \mathrm{CuSO}_{4}$ and $50 \mathrm{mM}$ 1-hydroxyproline at $\mathrm{pH} 4$ by MEKC. But it is worthy to note that the only CMC value of SDS determined by Chen et al..$^{22}$ might be considerably affected by the presence of $\mathrm{CuSO}_{4}$ and 1-hydroxyproline. Up to now, there is a general lack of data of CMC values of SDS in aqueous solution in the presence of acetonitrile and phosphate.

There are a number of methods which have been applied to the determination of CMC values of surfactants ${ }^{23}$. Most of the physicochemical property changes can be used for the determination of the $\mathrm{CMC}$, provided the measurements of particular property can be carried out accurately. Selection of a method to determine the CMC of a surfactant depends on structure and properties of the surfactant and the equipment availability in the experimenter's laboratory. Use of different methods may permit to obtain complementary and comparative results. This paper presents the determination of the CMC of SDS in aqueous solution in the presence of acetonitrile by conductometry and phosphate by fluorometry. The information provided in this paper is expected to be useful for optimization of separation and interpretation of migration behaviour of solutes in MEKC.

\section{EXPERIMENTAL}

Sodium dodecyl sulphate (SDS) (A.R.) was a product of Tianjin Bodi Chemical Holding Co. (Tianjin, China). Acetonitrile (A.R.) and sodium dihydrogen phosphate (A.R.) were purchased from Sinopharm Chemical Reagent Co.(Shanghai, China). Sodium hydrogen phosphate (A.R.) was purchased from Jiangsu Taicang No. 2 Chemical Factory (Taicang, China). Pyrene (99\%) was obtained from TCI (Shanghai, China). Double-distilled water was used throughout the experiments.

Solutions: The stock solution $(20 \mathrm{mM})$ of SDS was prepared in water. Working solutions were prepared by diluting the stock solution with appropriate quantities of water and/or acetonitrile to desired concentrations.

To study the effect of acetonitrile on the CMC of SDS, solutions of SDS were prepared in different volume ratios of acetonitrile to water, i.e., 0.5, 1, 2, 3, 4 and $5 \%$.

To study the effect of phosphate buffer on the CMC of SDS, solutions of SDS were prepared in acetonitrile-water $(3 \%, \mathrm{v} / \mathrm{v})$ binary solvent having different concentrations of phosphate ( $\mathrm{pH}=7.0)$, i.e., 10, 20, 30, 40 and $50 \mathrm{mM}$. Pyrene was first dissolved in acetonitrile and then was added to the above solution to obtain its concentration of $8 \times 10^{-3} \mathrm{mM}$ for fluorescence measurements.

For measurements of conductivity, a digital conductivity meter of Model DDS-11D and a platinum black electrode of Model of DJS-1C of Jiangsu Jiangfen Electoanalytical
Instrument Co. (Jiangyan, China) were used. Fluorescence measurements were carried out in a fluorescence spectrophotometer of Model RF-5301PC of Shimadzu (Kyoto, Japan). Fluorescent spectra were recorded in range of 344-480 nm with excitation at $334 \mathrm{~nm}$. The slit width was $5 \mathrm{~nm}$ for excitation and $3 \mathrm{~nm}$ for emission and the scan rate was $500 \mathrm{~nm} / \mathrm{min}$. Solutions for the measurements were kept at $30 \pm 0.1^{\circ} \mathrm{C}$ in a thermostatic water bath of Model W2200SP of Dayan Instrument Co. (Shanghai, China).

\section{RESULTS AND DISCUSSION}

Effect of acetonitrile on the CMC of SDS: The determination of the CMC of SDS by conductometry was made through plotting a graph of conductivity $\mathrm{k}$ as a function of SDS concentration. An abrupt change of slope marked the $\mathrm{CMC}$. The change in the conductance and conductivity at the CMC is due to the different degree of surfactant ionization below (surfactant monomers behave as strong electrolytes) and above (micelles are partially ionized) the $\mathrm{CMC}^{24}$. The conductivity of SDS at different concentrations in water-acetonitrile binary solvent under different volume ratios of acetonitrile to water was measured. Under each volume ratio of acetonitrile to water, the CMC of SDS was found by locating the intersection of the two straight lines obtained on plotting conductivity $v s$. concentration of SDS. Fig. 1 shows a typical plot of conductivity $v$ s. concentration of SDS, which was obtained in $3 \%(\mathrm{v} / \mathrm{v})$ acetonitrile to water.

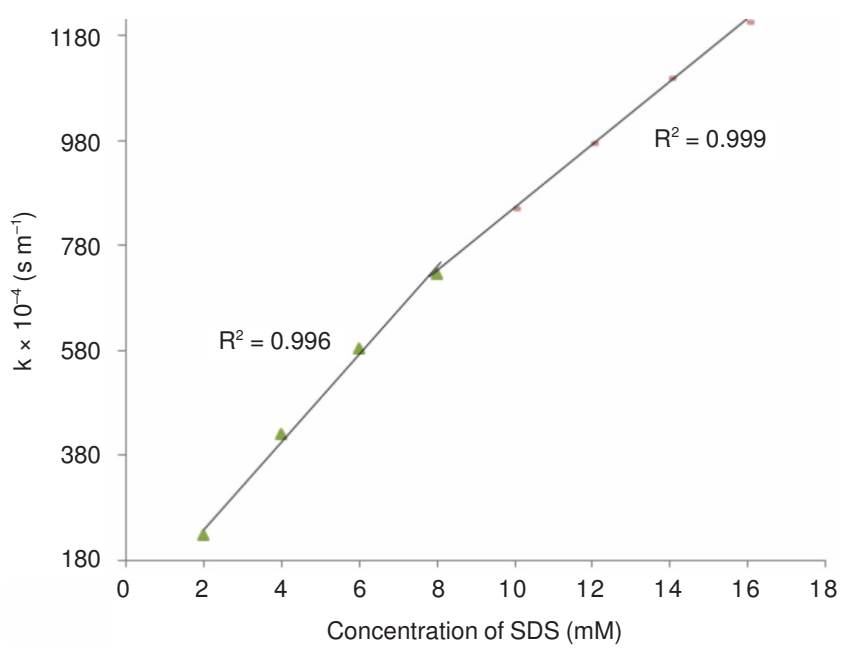

Fig. 1. Plot of conductivity $\mathrm{k} v s$. concentration of SDS in $3 \%(\mathrm{v} / \mathrm{v})$ acetonitrile water binary solvent

The effect of acetonitrile in the range of $0.5-5.0 \%(\mathrm{v} / \mathrm{v})$ to water on the CMC values of SDS, determined is shown in Fig. 2. The CMC values first decreased with increasing of the volume ratio of acetonitrile to water up to $3 \%$ and then increased. It is known that at a given temperature for a given surfactant only a certain amount of monomers can be accommodated in (a given volume of) solution and any further dissolution of the surfactant will be essentially transformed to micelles. Aggregation of ionic surfactants can occur if the interfacial energy available from the destruction of the nonpolar tail-solvent interfaces is greater than the work to be done against electrical repulsion of the ionic heads ${ }^{17}$. When acetonitrile 


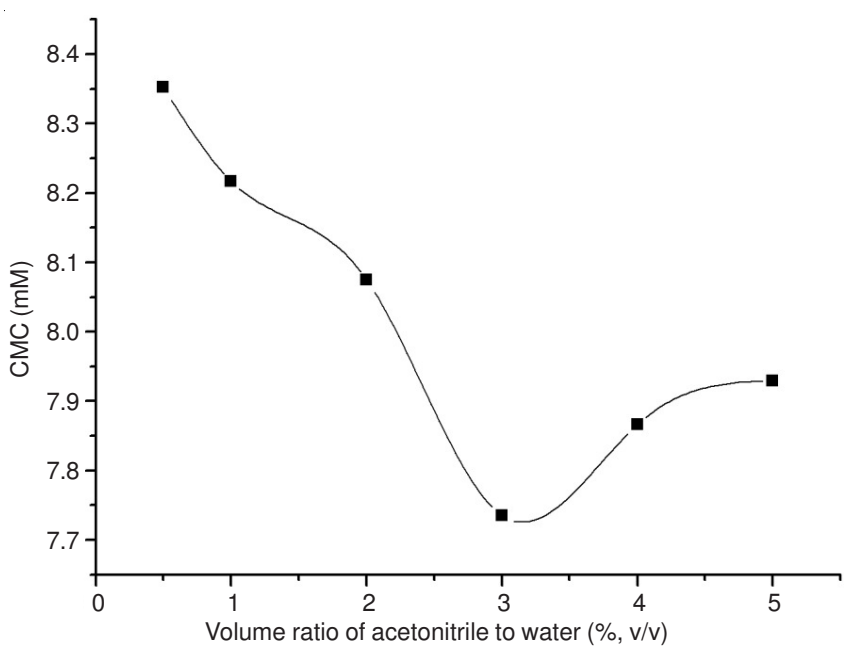

Fig. 2. Effect of acetonitrile on the CMC of SDS

was dissolved in water it would influence hydrophobic effect. At low concentrations of acetonitrile or low volume ratio of acetonitrile to water, the hydrophobic part of the surfactant was dissolved mainly interstitially and thus on adding SDS to the solution the surfactant monomers would coaggregate as the limit of interstitial mixing was attained, with the resultant formation of micelles at a lower concentration of SDS than in pure water. At high concentration of acetonitrile the structure of water was sufficiently disturbed that regular mixing of acetonitrile and water molecules became dominant so that when SDS was added to such a mixture it tended to dissolve regularly too, so that the driving force for micelle formation became weaker and micelles formed at a higher concentration of SDS. At intermediate concentrations of acetonitrile both tendencies occurred and when they were balanced a minimum, $7.7 \mathrm{mM}$, appeared in the $\mathrm{CMC}$ - acetonitrile concentration curve.

Effect of phosphate buffer on the CMC of SDS in acetonitrile-water binary solvent: We firstly determined the CMC of SDS under different concentrations of phosphate buffer at $\mathrm{pH} 7$ in acetonitrile-water $(3 \%, \mathrm{v} / \mathrm{v})$ binary solvent by conductometry. Conductometry worked well with concentration of phosphate below $30 \mathrm{mM}$. However, we were not able to unambiguously locate the intersection of the two curves obtained on plotting conductivity vs. concentration of SDS below and above the CMC when concentration of phosphate was above $30 \mathrm{mM}$. It was believed that it was the result of the large signal background generated by phosphate swamping the small difference in conductivity below and above the CMC of SDS. To determine the CMC of SDS in the presence of a broad range of concentration of phosphate we had to make use of other methods.

Determination of the CMC in aqueous solution by fluorometry involves the use of a hydrophobic fluorescence probe which exhibits different fluorescence characteristics depending upon the properties of the solubilizing medium. Pyrene is one of the few condensed aromatic hydrocarbons that show significant fine structure (vibrational bands) in their monomer fluorescence spectra in solution. The intensities of the various vibrational bands of pyrene show a strong dependence on solvent environment. The strong perturbation of the vibronic band intensities in pyrene monomer fluorescence spectra has been used for determining the CMC of SDS in aqueous solution $^{24-26}$. We applied this method to the determination of the CMC of SDS under different concentrations of phosphate at $\mathrm{pH} 7$ in acetonitrile-water (3\%, v/v) binary solvent.

Fig. 3 presents representative monomer fluorescence spectra for pyrene (in very dilute solutions, [pyrene] $=8 \times 10^{-3}$ $\mathrm{mM}$ ) under different concentration of SDS in unbuffered acetonitrile-water $(3 \%, \mathrm{v} / \mathrm{v})$ binary solvent. In the absence of micelles (below the CMC) pyrene sensed the polar environment of water and acetonitrile molecules; the ratio of fluorescence emission intensities corresponding to the first and third vibrational peaks $\left(\mathrm{I}_{\mathrm{I}} / \mathrm{I}_{\mathrm{III}}\right)$ (Fig. 3), was high. Above the CMC, when micelles were present and owing to the high hydrophobicity of pyrene molecules, these were solubilized in the interior micellar phase. That was a hydrocarbon-like solvent, so the environment sensed by pyrene was less polar and therefore the ratio $\mathrm{I}_{\mathrm{I}} / \mathrm{I}_{\mathrm{III}}$ decreased.

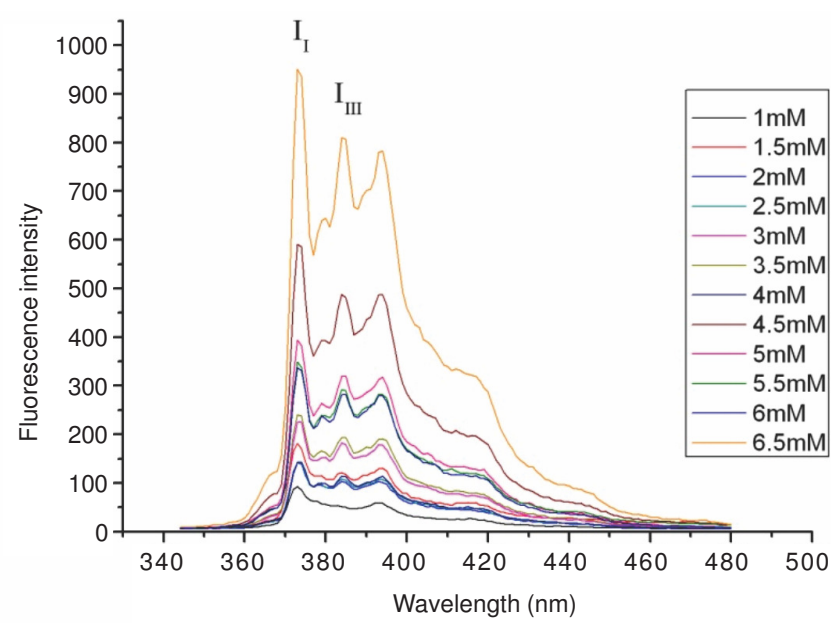

Fig. 3. Fluorescence spectra of pyrene in unbuffered acetonitrile-water $(3 \%, v / v)$ binary solvent under different concentrations of SDS

Fig. 4 shows the variation of the ratio of fluorescence emission intensities $\left(\mathrm{I}_{\mathrm{I}} / \mathrm{I}_{\mathrm{III}}\right)$ of pyrene as a function of the surfactant concentration of SDS in acetonitrile-water (3\%, v/v) binary solvent having different concentration of phosphate buffer (10-50 mM). The curves show a sharp decrease in the quotient intensities of the both vibrational peaks when micelles are formed (i.e., at the CMC). The CMC values of SDS corresponding to the surfactant concentrations at the sharp decrease are plotted in Fig. 5. The trend of the curve in Fig. 5 shows the effect of phosphate buffer on the CMC of SDS in acetonitrile-water (3\%, v/v) binary solvent. The CMC values of SDS decrease with increasing of the concentration of phosphate buffer. This trend agrees with the general observation that increasing electrolyte reduces the CMC of ionic surfactants ${ }^{18,27}$. The decrease in CMC with added electrolyte is due to a decrease in repulsive forces between the charged head-groups of the surfactants in the micelle as the counterion concentration in the ionic double layer is increased.

\section{Conclusion}

The effect of acetonitrile on the CMC of SDS was studied by conductometry. It was found that the CMC of SDS first 


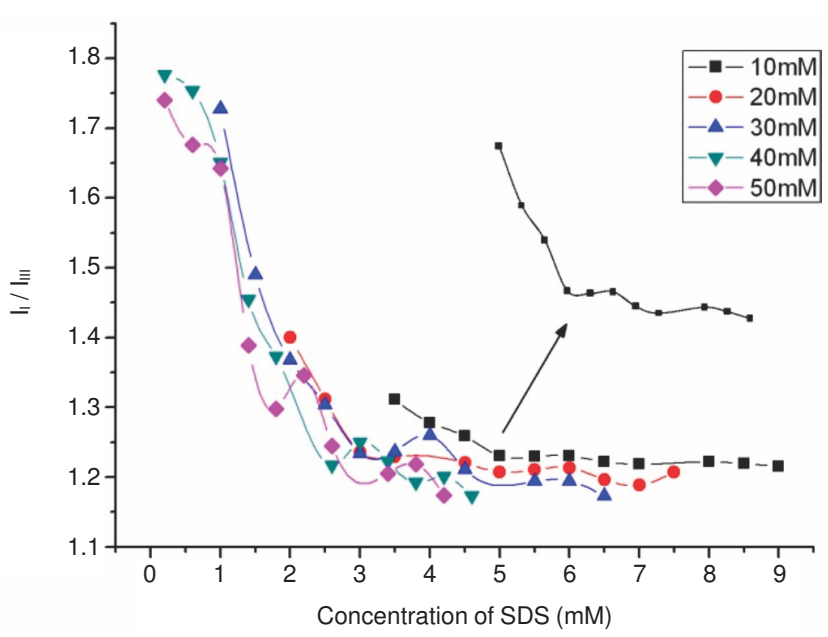

Fig. 4. Variation of the ratio of fluorescence emission intensities $\left(\mathrm{I}_{\mathrm{I}} / \mathrm{I}_{\mathrm{III}}\right)$ of pyrene as a function of the surfactant concentration of SDS in acetonitrile-water $(3 \%, \mathrm{v} / \mathrm{v})$ binary solvent buffered at $\mathrm{pH} 7$ with phosphate $(10-50 \mathrm{mM})$

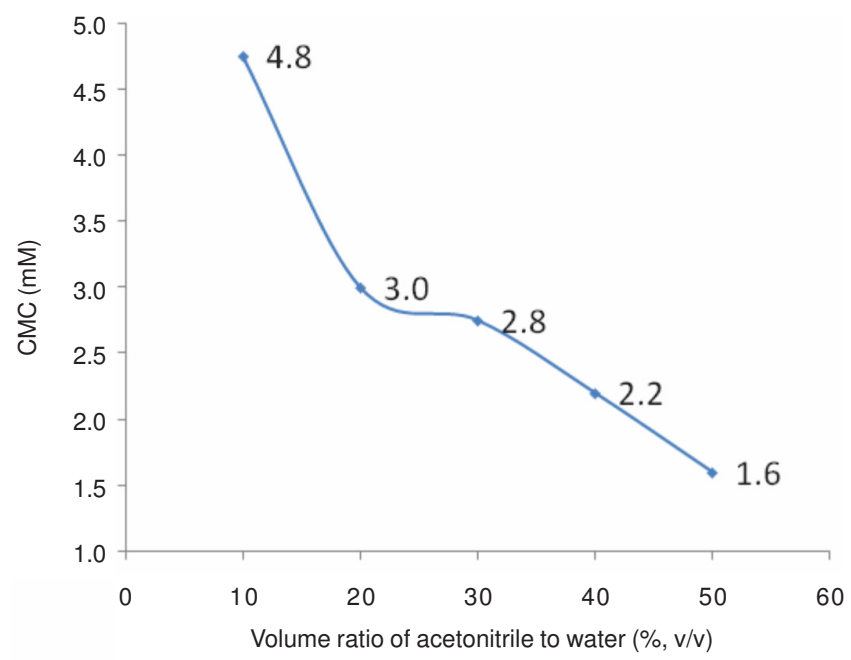

Fig. 5. Effect of concentration of phosphate buffer on the CMC of SDS in acetonitrile-water $(3 \%$, v/v) binary solvent

decreased up to $3 \%(\mathrm{v} / \mathrm{v})$ and then increased with increasing of the volume ratio of acetonitrile to water up to $5 \%(\mathrm{v} / \mathrm{v})$. The minimum of the CMC of SDS in acetonitrile-water binary solvent was found at the volume ratio of $3 \%, \mathrm{v} / \mathrm{v}$, acetonitrile to water and determined to be $7.7 \mathrm{mM}$. The effect of phosphate buffer on the CMC of SDS in acetonitrile-water $(3 \%, \mathrm{v} / \mathrm{v})$ binary solvent was studied by fluorometry with the use of pyrene as a probe. The CMC value of SDS decreased with increasing of phosphate in the concentration range of 10-50 $\mathrm{mM}$.

\section{ACKNOWLEDGEMENTS}

The work was supported by Shanghai Municipal Education Commission (Grant No. 08YZ06).

\section{REFERENCES}

1. G.L. McIntire and J.G. Dorsey, Crit. Rev. Anal. Chem., 21, 257 (1990).

2. E. Pramauro and E. Pelizzetti, Surfactants in Analytical Chemistry: Applications of Organized Amphiphilic Media, Elsevier, Amsterdam (1996).

3. A. Ballesteros-Gomez, M.D. Sicilia and S. Rubio, Anal. Chim. Acta, 677, 108 (2010)

4. S. Terabe, K. Otsuka, K. Ichikawa, A. Tsuchiya and T. Ando, Anal. Chem., 56, 111 (1984)

5. S. Terabe, Anal. Chem., 76, 240A (2004).

6. H. Watanabe and H. Tanaka, Talanta, 25, 585 (1978).

7. F.H. Quina and W.L. Hinze, Ind. Eng. Chem. Res., 38, 4150 (1999).

8. P.G. Muijselaar, K. Otsuka and S. Terabe, J. Chromatogr. A, 780, 41 (1997).

9. M. Silva, Electrophoresis, 32, 149 (2011).

10. L.T.H. Duong, J.C. Kraak and W. Th. Kok, J. Pharm. Biomed. Anal., 22, 879 (2000).

11. T.M.H. Choy, W.H. Chan, A.W.M. Lee and C.W. Huie, Electrophoresis, 24, 3116 (2003).

12. T.S.K. So, L. Jia and C.W. Huie, Electrophoresis, 22, 2159 (2001).

13. U. Butehorn and U. Pyell, J. Chromatogr. A, 792, 157 (1997).

14. R.M. Seifar, J.C. Kraak and W.T. Kok, Anal. Chem., 769, 2772 (1999).

15. U. Butehorn and U. Pyell, J. Chromatogr. A, 772, 27 (1997).

16. S. Terabe, J. Pharm. Biomed. Anal., 10, 705 (1992).

17. A.F.H. Ward, Proc. R. Soc. London, Ser. A, 176, 421 (1940).

18. M.L. Corrin and W.D. Harkins, J. Am. Chem. Soc., 69, 683 (1947).

19. I. Benito, M.A. Garcia, C. Monge, J.M. Saz and M.L. Marina, Colloid. Surf. A, 125, 221 (1997).

20. E. Fuguet, C. Rafols, M. Roses and E. Bosch, Anal. Chim. Acta, 548, 95 (2005).

21. J.C. Jacquier and P.L. Desbene, J. Chromatogr. A, 743, 307 (1996).

22. Z. Chen, J.-M. Lin, K. Uchiyama and T. Hobo, Anal. Chim. Acta, 403, 173 (2000)

23. K. Shinoda, Colloidal Surfactants: Some Physicochemical Properties, Academic press, New York (1963).

24. A. Domínguez, A. Fernández, N. González, E. Iglesias and L. Montenegro, J. Chem. Educ., 74, 1227 (1997).

25. K. Kalyanasundaram and J.K. Thomas, J. Am. Chem. Soc., 99, 2039 (1977).

26. K.P. Ananthapadmanabhan, E.D. Goddard, N.J. Turro and P.L. Kuo, Langmuir, 1, 352 (1985).

27. H.B. Klevens, J. Phys. Chem., 52, 130 (1948). 